\title{
A Review on Brain Tumor Segmentation and Definition using Soft Computing Technique
}

\author{
Ms.Pooja S. Deshpande ${ }^{1}$, Mr. Shikant Honade ${ }^{2}$ \\ ${ }^{1}$ (Electronics \& Telecommunication, SGBAU, India) \\ ${ }^{2}$ (Electronics \& Telecommunication, SGBAU, India)
}

\begin{abstract}
Brain tumor is most severe disease; most of populations in world affected due to brain tumor. Now day death rate because brain tumor gradually increases. For that consideration, most prominent method implemented for brain tumor detection and segmentation. When most normal cells grow, old cells die or damaged and new cells take their place. Sometimes this process goes wrong. New cells form when the body does not need them, and old or damaged cell do not die as they should.The buildup of extras cells often forms a mass of tissue called a growth or tumor. Earlier detection, diagnosis and proper treatment of brain tumor are essential to prevent human death. An effective brain tumor detection and segmentation using MR image is an essential task in medical field.

A number of research papers related to medical image segmentation methods are studied. Segmentation and detection plays an important role in the processing of medical images. There are various segmentation methods implemented for brain tumor detection. These methods include $k$-means clustering with watershed segmentation algorithm; optimized k-means clustering with genetic algorithm and optimized c-means clustering with genetic algorithm. Traditional k-means algorithm is sensitive to the initial cluster centers. Genetic c-means and kmeans clustering techniques used to detect tumor in MRI of brain images etc.

The proposed work deals with the use of soft computing technique for brain tumor detection and segmentation using MRI images. The soft computing technique gives more improved parameter.
\end{abstract}

Keywords: MRI, brain tumor, Segmentation, Detection

\section{Introduction}

The properties of problem of semi-automatic tumor detection make it an excellent research challenge in the fields of medical image analysis and pattern recognition, in general. The main motivations for this investigated effort are as follows:

- The manual segmentation by qualified professionals has two major drawbacks. The first drawback is that producing manual segmentations is extremely time consuming, with higher accuracies more finely detailed volumes demanding increased time from medical experts .

- Accurate segmentation methods may possibly also lead to new applications, including effective content based image retrieval in large medical databases. This could allow clinicians to find the similar images in historical data based on tumor location, size, similar pattern of growth, or a variety of other factor.

In medical field accuracy and delay time plays vital role in every aspect of technology to achieve, access and accommodated desired response of system. The most eminent field of technology, which completely enhance and fulfills the primary need of society is proper diagnoses. Although after implementing verities of invention research in segmentation and detection of brain tumor ; inventors are unable to develop system with high accuracy, With this unfortunate truth in medical field to provide Proper diagnosis is biggest challenge for inventors.

With this motivation being an engineer, propose paper to develop system of brain tumor segmentation and detection which would provides better performance parameters.

Tumor defined as the abnormal growth of the tissues. Brain tumor is an abnormal mass of tissue in which cells grow and multiply uncontrollably, seemingly unchecked by the mechanisms that control normal cells. Brain tumors can be primary or metastatic, and either malignant or benign.

Magnetic Resonance Imaging (MRI) is an advanced medical imaging technique used to produce high quality images of the parts contained in the human body MRI imaging is often used when treating brain tumors, ankle, and foot. From these high-resolution images, we can derive detailed anatomical information to examine human brain development and discover abnormalities.

Segmenting brain tumors is a very difficult task. In the first place, there are a large class of tumor types, which have a variety of shapes and sizes. Appearance of brain tumors different locations in the brain with different image intensities is another factor that makes difficult automated brain tumor detection and segmentation. The intensity values seen on an MRI scan for a particular brain depends primarily on the content of that pixel versus neighboring tissue and on other factors including the presence of abnormality. In normal 
brain MR images intensity level of brain tissues in the order of increasing brightness is cerebrospinal fluid (CSF), gray matter (GM), white matter (WM) in T1-weighted (T1-w) and WM, GM and CSF in T2- weighted (T2-w) image. In tumorous brain MR images intensity level of tumorous tissues exhibit different intensity level on T1-w and T2-w images based on the type of tumor. On T1-w most tumors have low or intermediate signal intensity but for some tumors this does not hold true, for example, glioblastoma multiforme tumor has high signal intensity. On T2-w most tumors have bright intensity but there are tumors which have low intensity, the classic examples are lymphoma tumors.

Segmenting brain tumors is a very difficult task. In the first place, there are a large class of tumor types, which have a variety of shapes and sizes. Appearance of brain tumors different locations in the brain with different image intensities is another factor that makes difficult automated brain tumor detection and segmentation. The intensity values seen on an MRI scan for a particular brain depends primarily on the content of that pixel versus neighboring tissue and on other factors including the presence of abnormality. The main objective of the present work is segmentation method is to detect and extract the tumor region in MRI images.

\section{Related Research}

A number of research papers related to medical image segmentation and detection method were studied. A report of the literature survey is presented here. Ishmam Zabir et.al, demonstrated automatic system of Brain Tumor Detection and Segmentation based on Multi-Modal MRI Images Based on Region Growing and Level Set Evolution. Glioma is a type of brain tumor, originates from glial cells. Approximately $80 \%$ of them are malignant. In this document, the segmented area obtained from the conventional region-growing approach is automatically selecting as the initial contour to the iterative distance regularized level set evolution method thus removing the need of selecting the initial region of interest by the user. Therefore, a computer aided fully automated technique developed to detect glioma from multimodal MRI images \& segment the tumor region from whole image [1].

Praveen et.al, correspond to detection method of brain tumor in MRI Images, using Combination of Fuzzy C-Means and SVM. MRI is the most important technique, in detecting the brain tumor. In the paper data, mining methods used for classification of MRI images. A new hybrid technique based on the support vector machine (SVM) and fuzzy c-means for brain tumor classification is proposed. The algorithm is a combination of support vector machine (SVM) and fuzzy c-means, a hybrid technique for prediction of brain tumor. In this algorithm, the image enhanced using enhancement techniques such as contrast improvement, and mid-range stretch. Double thresholding and morphological operations used for skull striping. Fuzzy c-means (FCM) clustering used for the segmentation of the image to detect the suspicious region in brain MRI image [2].

I. Kailash Sinha et.al, published paper efficient Segmentation Methods for Tumor Detection in MRI images. This document presents a comparative study of three segmentation methods implemented for tumor detection. The methods include k-means clustering with watershed segmentation algorithm, optimized k-means clustering with genetic algorithm and optimized c- means clustering with genetic algorithm. Traditional k-means algorithm is sensitive to the initial cluster centers. Genetic $\mathrm{c}$ means and k-means clustering techniques used to detect tumor in MRI of brain images [3].

Sarah Parisot et.al, was proposed conception based on Graph-based Detection, Segmentation \& Characterization. In this manuscript proposed a novel approach for detection, segmentation and characterization of brain tumors. This method exploits prior knowledge in the form of a sparse graph representing the expected spatial positions of tumor classes. Such information coupled with image based classification techniques along with spatial smoothness constraints towards producing a reliable detection map within a unified graphical model formulation. Efficient linear programming both in terms of performance as well as in terms of computational load considered to recover the lowest potential of the objective function [5]. These are the methods used for the implementation for the brain tumor detection and segmentation. The proposed report gives the most efficient but the fastest method for the brain tumor detection and segmentation, which gives the parameters. This proposed paper has the easiest step of image processing. The method easily implemented simply by using MATLAB tool with the simple image processing toolbox.

\section{Proposed Method}

The proposed paper demonstrates that the efficient method could successfully detect the brain tumor and help the doctors for analyzing tumor percentage and region. To overcome the above used soft computing technique. The performance of soft computing technique is evaluated basis of parameters under consideration like delay time, percentage of tumor, mean, standard deviation, entropy, sensitivity, specificity, accuracy and FPR.

The inventor of fuzzy logic, Lotfi A. Zadeh, proposed the term soft computing. Describes it as follows "Soft computing is a collection of methodologies that aim to exploit the tolerance for imprecision and uncertainty to achieve tractability, robustness, and low solution cost. Its principal constituents are fuzzy logic, 
neuron computing, and probabilistic reasoning. Soft computing is likely to play an increasingly important role in many application areas, including software engineering. The role model for soft computing is the human mind. Soft Computing is the fusion of methodologies that designed to model and enable solutions to real world problems, which are not modeled or too difficult to model, mathematically.Soft computing optimization technique to find solution of problems, which are very hard to answer. Soft computing not precisely defined. It consists of distinct concepts and techniques that aim to overcome the difficulties encountered in real world problems. These problems result from the fact that our world seems to be imprecise, uncertain and difficult to categorize. For example, the uncertainty in a measured quantity is due to inherent variations in the measurement process itself. The uncertainty in a result is due to the combined and accumulated effects of these measurement uncertainties, which used in the calculation of that result. In many cases, a lot of work and cost can achieve the increase in precision and certainty. Zadeh gives as an example the travel salesperson problem, in which the computation time is a function of accuracy and it increases exponentially.Another possible definition of soft computing is to consider it as an anti-thesis to the concept of computer we now have, which described with all the adjectives such as hard, crisp, rigid, inflexible and stupid. Along this track, one may see soft computing as an attempt to mimic natural creatures: plants, animals, human beings, which are soft, flexible, adaptive and clever. In this sense, soft computing is the name of a family of problem-solving methods that have analogy with biological reasoning and problem solving (sometimes referred to as cognitive computing). The basic methods included in cognitive computing are fuzzy logic, neural networks and genetic algorithms the methods that do not derive from classical theories. Fuzzy logic is mainly associated to imprecision, approximate reasoning and computing with words, Neuro computing to learning and curve fitting (also to classification), and probabilistic reasoning to uncertainty and belief propagation (belief networks). These methods have in common that are

1. are nonlinear,

2. have ability to deal with non-linearities,

3. follow more human-like reasoning paths than classical methods,

4. utilize self-learning,

5. utilize yet-to-be-proven theorems,

6. are robust in the presence of noise or errors.

"Soft Computing is not just a mixture of these ingredients, but a discipline in which each constituent contributes a distinct methodology for addressing problems in its domain, in a complementary rather than competitive way."

\section{Conclusion}

Magnetic Resonance Imaging (MRI) is an important examination and diagnosis method for brain tumors in medical imaging. With a sound mechanism and clear imaging of soft tissues, the doctor on the patient's diagnosis can be scientific and rational, to grasp the exact progression of the disease state, which would set out the appropriate treatment, surgery and following-up to a series of disease control measures. Computeraided analysis is to reduce the workload of doctors, to improve the diagnostic accuracy of the paramedical analysis, and meanwhile to improve the automatic degree in practice.

\section{Reference}

[1]. Ishmam Zabir, Sudip Paul et.el, "Automatic Brain Tumor Detection and Segmentation from Multi-Modal MRI Images Based on Region Growing and Level Set Evolution" IEEE International Confererance on Electrical and Computer Engg., Dhaka , Bangladesh, 2015, PP. 503-506.

[2]. Praveen and Amritpal Singh "Detection of Brain Tumor in MRI Images, using Combination of Fuzzy C-Means and SVM", IEEE, Dept. of computer science and engineering ,Govt. women engineering college Ajmer, India, 2015, PP.98 -101.

[3]. Kailash Sinha1, G.R.Sinha , "Efficient Segmentation Methods for Tumor Detection in MRI Images", IEEE, Student's Conference on Electrical, Electronics and Computer Science,ShriShankaracharya Technical Campus Bhilai, India, 2014, PP. 1-6.

[4]. J.Vijay,J.Subhashini“An Efficient Brain Tumor Detection Methodology Using K-Means Clustering Algorithm”,IEEE, International conference on Communication and Signal Processing, Chennai, India, 2013, PP.653-657.

[5]. Sarah Parisot, Hugues Duffau, St'ephane Chemouny, "Graph-based Detection, Segmentation \& Characterization of Brain Tumors", IEEE, Center for Visual Computing, Ecole Centrale de Paris, France, 2013, PP.988-995.

[6]. Atiq Islam, Syed M. S. Reza and Khan M. Iftekharuddin, "Multi-fractal Texture Estimation for Detection and Segmentation of Brain Tumors" Department of Electrical and Computer Engineering, Old Dominion University, Norfolk, VA 23529, USA, 2013.

[7]. K.S.Tamilselvan, Dr.G.Murugesan and B.Gnanasekaran, "Brain Tumor Detection from Clinical CT and MRI Images using WTFCM Algorithm" Department of ECE Velalar College of Engineering and Technology, Erode, Anna University, India, 2013, PP.260-263.

[8]. M. Usman Akram and Anam Usman,“Computer Aided System for Brain Tumor Detection and Segmentation”, ,IEEE ,Department qf Computer and Software Engineering, Bahria University, Islamabad, Pakistan , 2011,PP.299-302.

[9]. Ms.T.K. Sreeja and Ashmi Mohammed "A Skull-Stripping Method Based on Modified Morphological Processing", International Conference on Signal Processing, Communication, Computing and Networking Technologies, IEEE, Noorul Islam University Kumaracoil, Tamil Nadu,India,2011,PP.313-316.

[10]. Gayatri Mirajkar, Balaji Barbadekar, "Automatic Segmentation of Brain Tumors from MR Images using Undedicated Wavelet Transform and Gabor Wavelets" 2010 IEEE, PP. 648-652.

[11]. Xie Mei, Zhen Zheng, Wu Bingrong, Li Guo, “The Edge Detection of Brain Tumor” School of Electronic Engineering,University of Electronic Science and Technology of China Chengdu,IEEE, Sichuan, China, 2009, PP 477-479. 
[12]. D.Jude J Hemanth, D.Selvathi and J.Anithal, "Effective Fuzzy Clustering Algorithm for Abnormal MR Brain Image Segmentation",IEEE International Advance Computing Conference (IACC 2009), Karunya University, Coimbatore Mepco Schlenk Engineering College, Sivakasi, India, 2009, PP.609-614

[13]. Jehan Zeb Shah, Syed Afaq Husain " A fuzzy based Adaptive BPNN Learning Algorithm for Segmentation of thevBrain MR images" University of' Techno logy ,IEEE,Malaysia, SZABIST Is+, Flamahad, 2004, PP.86-90.

[14]. A. S. Capelle, 0. Alata, C. Femandez, S. Lefevre "Unsupervised Segmentation for Atomatic Detection of brain tumors in MRI ,Univ. Poitiers, Bat., Futuroscope Chasseneuil Cedex, FRANCE,IEEE,2004,PP. 613-613.

[15]. Mangipudi Partha Sarathi, Mohammed Ahmed Ansari, Vaclav Uher, RadimBurget, and Malay Kishore Dutta “Automated Brain Tumor Segmentation using Novel Feature Point Detector and Seeded Region Growing” IEEE,2013, PP. 648-652.

[16]. N. HemaRajini , R.Bhavani, "Enhancing K-means and Kernelized Fuzzy C-means Clustering with Cluster Center Initialization in Segmenting MRI brain images" Department of Computer Science and Engineering, Annamalai University, AnnamalaiNagar, Tamilnadu, , IEEE, 2011, PP. 259-26.

[17]. Sindhumol S., Anil Kumor and Kannan Balakrishnam, "Wavelet based Indepentent Component Analysis for Multispectral Brain Tissue Classification”, Dept.of computer application, kochi, India, IEEE, 2013, PP.415-418.

[18]. Xin-She Yang, "Firefly Algorithms for Multimodal Optimization", Department of Engineering, University of Cambridge, Trumpington Street, Cambridge, PP 169-179.

[19]. Xin-She Yang "Firefly Algorithm: Recent Advances and Applications", School of Science and Technology, Middlesex University, The Burroughs, London.

[20]. Dmitry bystrov "lecture notice introduction to soft computing"Henry Han, Xiao-Li Li,"Multi-resolution independent component analysis for high- performance tumor classification and biomarker discovery, BMC Bioinformatics", 2011.

[21]. Abdullah N, Lee Wee Chuen, Ngah U K, Ahmad K A, "Improvement of MRI brain classification using principal component analysis", Proc IEEE IntConf on Control System, Computing and Engineering (ICCSCE), 2011, 557-561.

[22]. Eyal E, Bloch B N, Rofsky N M, Furman-Haran E, Genega E M, Lenkinski R E, DeganiH.,"Principal Component Analysis for Dynamic Contrast-Enhanced MRI in Human Prostate Cancer, Invest Radiol”, 2010, PP. 174-181. 